\title{
Formula for Two-Carrier Intermodulation Distortion in Wavelength Converted Subcarrier Multiplexed Signals via Cross Gain Modulation
}

\author{
José Capmany, Senior Member, IEEE, Eva Peral, Member, IEEE, and Daniel Pastor, Member, IEEE
}

\begin{abstract}
We present, for the first time to our knowledge, closed expression for the computation of the harmonic and intermodulation distortions that appear on a wavelength converted Two-tone subcarrier modulation (SCM) signal via cross gain modulation.
\end{abstract}

\section{INTRODUCTION}

W AVELENGTH conversion is a key technology for the implementation of future flexible WDM networks [1]. Among the possible technologies for its implementation, the use of the semiconductor optical amplifiers (SOA's) under nonlinear operation has attracted considerable attention, leading to the successful demonstration of both cross-gain (XGM) and cross-phase (XPM) modulation schemes [2] in high bit rate digital channels.

Another interesting application of these technologies is the optical frequency translation of analogue or subcarrier multiplexed (SCM) signals [3], [4]. Schemes based on four-wave mixing (FWM) [3] and XGM [4] in SOA's have been experimentally demonstrated. The former has the advantage of providing signal transparency at the cost of low conversion efficiency, whereas the later provides a bit rate/frequency limited transparency and a high efficiency.

The abovementioned transparency limitations in XGM-based wavelength converters stem from the limited bandwidth of the conversion transfer function and are well understood when dealing with digital signals [2], [5]. There is however, at least to the authors' knowledge, not such a clear picture when analogue SCM signals are considered, since the harmonic and intermodulation distortion arising from the nonlinear interaction between photons and electrons in the SOA has to be added to the inherent XGM bandwidth limitation.

The purpose of this letter is to provide closed formulas for the harmonic and intermodulation distortion that appear on a wavelength converted two-tone SCM signal via XGM. These expressions can be useful for the design of systems and networks where wavelength-division multiplexing (WDM) channels carrying SCM signals experience wavelength conversion.

Manuscript received July 16, 1999; revised November 23, 1999. This work was supported by the Spanish Government (CICYT-TIC98-0346) and the European Comission (COST 267).

J. Capmany and D. Pastor are with the Grupo de Comunicaciones Opticas, Departamento de Comunicaciones, Universidad Politécnica de Valencia, 46022 Valencia, Spain.

E. Peral was with the Department of Applied Physics, California Institute of Technology, Pasadena, CA 91125 USA. She is now with Ortel Corporation, Alhambra, CA 91803 USA.

Publisher Item Identifier S 1041-1135(00)01963-7.

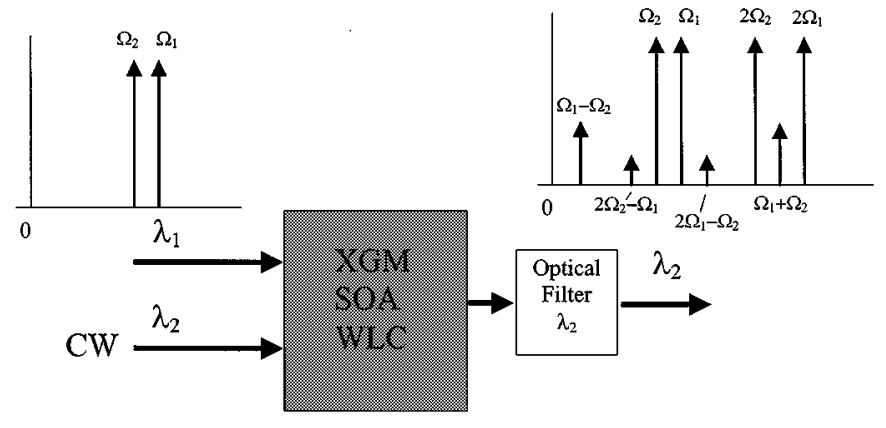

Fig. 1. Intermodulation distortion of a SCM signal composed of two modulated tones arising from its wavelength conversion via XGM in a SOA.

\section{DERIVATION OF THE FORMULA}

Fig. 1 shows the problem under consideration. The rate equations describing the propagation of two optical fields in a lossless waveguide were derived in [5]. We assume that the frequency detuning between the copropagating pump and probe fields is larger than the reciprocal of the lifetime of the excited carriers divided by $2 \pi$ to guarantee that four wave mixing products due to the beating between them are negligible. Under this assumption the rate equations can be expressed for the general case of $M$ fields of intensity $P_{i}$ (in $\left.\mathrm{mW}\right), i=1 \ldots M$, at wavelengths $\lambda_{i}$ as

$$
\begin{aligned}
P_{i}(t, z)=P_{i}(t, 0) G_{i} \exp \left[\Gamma a_{i}\left(\sigma-\sigma_{s}\right)\right] \\
\frac{d \sigma}{d t}=-\left(\frac{\sigma-\sigma_{o}}{\tau_{s}}\right) \\
-\sum_{i} C_{i} P_{i}(t, 0)\left[G_{i} \exp \left[\Gamma a_{i}\left(\sigma-\sigma_{s}\right)\right]-1\right]
\end{aligned}
$$

where

$$
\sigma(t, z)=\int_{0}^{z} N\left(t, z^{\prime}\right) d z^{\prime}
$$

Here, $\sigma(t, z)$ represents the spatially integrated (from $z^{\prime}=0$ to $\left.z^{\prime}=z\right)$ carrier density, $G_{i}=\exp \left[\Gamma a_{i}\left(\sigma_{s}-N_{i} z\right)\right]$ is the saturated gain for mode $i ; \sigma_{s}$ is the spatially integrated $\mathrm{CW}$ (steady-state) carrier density, $a_{i}$ the differential gain at wavelength $\lambda_{i}, \sigma_{0}=\tau_{s}(I / \mathrm{eV}) z$, is the transparency integrated carrier density, with $I$ the current, $V$ the active volume, and $\tau_{s}$ the carrier lifetime. The constant $C_{i}=1 /\left(\Gamma \hbar \omega_{0} A_{\mathrm{eff}_{i}}\right)$ is related 
to the saturation power defined as $P_{\mathrm{sat}_{i}}=1 /\left(\Gamma C_{i} a_{i} \tau_{s}\right)=$ $A_{\mathrm{eff}_{i}} \hbar \omega_{o} / a_{i} \tau_{s}$ with $A_{\mathrm{eff}_{i}}$ the effective mode area and $\Gamma$ the mode confinement factor.

In our analysis, we assume that: a) The input pump signal centred at $\lambda_{2}$ is continuous-wave (CW), i.e., $\left.P_{2}(t, 0)=\bar{P}_{20} ; \mathrm{b}\right)$ The probe signal, carrying the SCM band is centered at $\lambda_{1}$ and is composed of two RF tones with equal modulation index $m$ placed at frequencies $\Omega_{1}$, and $\Omega_{2}$, i.e.,

$$
\begin{aligned}
P_{1}(t, 0)= & \bar{P}_{10}+\delta p_{10}=\bar{P}_{10}\left(1+m\left(\Omega_{1}\right) e^{j \Omega_{1} t}\right. \\
& \left.+m\left(-\Omega_{1}\right) e^{-j \Omega_{1} t}+m\left(\Omega_{2}\right) e^{j \Omega_{2} t}+m\left(-\Omega_{2}\right) e^{j \Omega_{2} t}\right)
\end{aligned}
$$

c) A perturbative approach up to the third order for the determination of the spatially integrated carrier density similar to that of [6] and [7] is assumed i.e., $\sigma=\sigma_{s}+\delta \sigma_{1}+\delta \sigma_{2}+\delta \sigma_{3}$, where the subscript " $i$ " in $\delta \sigma_{i}$ accounts for the order of the perturbation term so

$$
\begin{aligned}
& \delta \sigma_{1}=\sum_{i=1}^{2} \Delta \sigma\left( \pm \Omega_{i}\right) e^{ \pm j \Omega_{i} t} \\
& \delta \sigma_{2}=\sum_{j=1}^{2} \sum_{i=1}^{2} \Delta \sigma\left( \pm \Omega_{i} \pm \Omega_{j}\right) e^{j\left( \pm \Omega_{i} \pm \Omega_{j}\right) t}
\end{aligned}
$$

and so on. With the above considerations, (2) yields one algebraic equation for $\sigma_{s}$ and three differential equations for $\delta \sigma_{i}$ $i=1,2,3$, which can be solved successively, i.e., starting with $\delta \sigma_{1}$ and using this value to obtain $\delta \sigma_{2}$ and so on

$$
\begin{aligned}
0= & -\left(\sigma_{s}-\sigma_{0}\right)-\left(\frac{\bar{P}_{10}}{\Gamma a_{1} P_{\text {sat } 1}}\right)\left(e^{\Gamma a_{1}\left(\sigma_{s}-N_{1} z\right)}-1\right) \\
& -\left(\frac{\bar{P}_{20}}{\Gamma a_{2} P_{\text {sat } 2}}\right)\left(e^{\Gamma a_{2}\left(\sigma_{s}-N_{2} z\right)}-1\right) \\
\frac{d \delta \sigma_{1}}{d t}= & -\frac{\delta \sigma_{1}}{\tau_{\text {eff }}}-C\left(G_{1}-1\right) \delta p_{10} \\
\frac{d \delta \sigma_{2}}{d t}= & -\frac{\delta \sigma_{2}}{\tau_{\text {eff }}}-C G_{1} \Gamma a_{1} \delta p_{10} \delta \sigma_{1}-\frac{\Xi_{1}}{2 \tau_{s 1}} \delta \sigma_{1}^{2} \\
\frac{d \delta \sigma_{3}}{d t}= & -\frac{\delta \sigma_{3}}{\tau_{\text {eff }}}-C G_{1} \Gamma a_{1} \delta p_{10} \delta \sigma_{2} \\
& -\frac{C G_{1} \Gamma^{2} a_{1}^{2} \delta p_{10} \delta \sigma_{1}^{2}}{2 \tau_{s 1}}-\frac{\Xi_{1} \delta \sigma_{1} \delta \sigma_{2}}{\tau_{s 1}}-\frac{\Xi_{2}}{6 \tau_{s 1}} \delta \sigma_{1}^{3}
\end{aligned}
$$

where

$$
\begin{aligned}
\Xi_{1} & =\Gamma a_{1}\left(C \Gamma a_{1} \bar{P}_{10} G_{1}+C \Gamma a_{2} \bar{P}_{20} G_{2}\left(\frac{a_{2}}{a_{1}}\right)\right) \\
& =\Gamma a_{1} \tau_{s 1}\left(\frac{1}{\tau_{s 1}}+\frac{1}{\tau_{s 2}}\left(\frac{a_{2}}{a_{1}}\right)\right) \\
\Xi_{2} & =\Gamma^{2} a_{1}^{2}\left(c \Gamma a_{1} \bar{P}_{10} G_{1}+C \Gamma a_{2} \bar{P}_{20} G_{2}\left(\frac{a_{2}}{a_{1}}\right)^{2}\right) \\
& =\Gamma^{2} a_{1}^{2} \tau_{s 1}\left(\frac{1}{\tau_{s 1}}+\frac{1}{\tau_{s 2}}\left(\frac{a_{2}}{a_{1}}\right)^{2}\right) \\
\frac{1}{\tau_{\text {eff }}} & =\frac{1}{\tau_{s}}+\frac{1}{\tau_{s 1}}+\frac{1}{\tau_{s 2}}
\end{aligned}
$$

and the approximation $\exp (x)-1 \approx x+x^{2} / 2 !+x^{3} / 3$ ! has been employed. $\tau_{s i}=A_{\text {effi }} \hbar \omega_{o} /\left(a_{i} \bar{P}_{i}\right) i=1,2$ are the lifetimes for the stimulated recombination due to the field powers [5].
The solution of (4) yields the following results for the harmonic distortion, second-, and third-order intermodulation products of the spatially integrated carrier density

$$
\begin{aligned}
\Delta \sigma( \pm \Omega)= & \left(\frac{\tau_{\text {eff }}}{\tau_{s 1}}\right) \frac{m}{\Gamma a_{1}}\left(\frac{G_{1}-1}{G_{1}}\right) H\left( \pm \Omega_{i}\right) \\
\Delta \sigma\left( \pm 2 \Omega_{i}\right)=\left(\frac{\tau_{\text {eff }}}{\tau_{s 1}}\right) H\left( \pm 2 \Omega_{i}\right) & \cdot\left[m\left( \pm \Omega_{i}\right) \Delta \sigma\left( \pm \Omega_{i}\right)+\frac{\Xi_{1} \Delta \sigma^{2}\left( \pm \Omega_{i}\right)}{2}\right] \\
\Delta \sigma\left(\Omega_{1} \pm \Omega_{2}\right)=\left(\frac{\tau_{\text {eff }}}{\tau_{s 1}}\right) H\left(\Omega_{1} \pm \Omega_{2}\right)\left[\left(m\left(\Omega_{1}\right) \Delta \sigma\left( \pm \Omega_{2}\right)\right.\right. & \left.\left.+\Omega_{2}\right) \Delta \sigma\left(\Omega_{1}\right)\right) \\
& \left.+\Xi_{1} \Delta \sigma\left(\Omega_{1}\right) \Delta \sigma\left( \pm \Omega_{2}\right)\right] \\
\Delta \sigma\left(2 \Omega_{1}-\Omega_{2}\right)= & \left(\frac{\tau_{\text {eff }}}{\tau_{s 1}}\right) H\left(2 \Omega_{1}-\Omega_{2}\right) \\
& \cdot\left\{\left[m\left(-\Omega_{2}\right) \Delta \sigma\left(2 \Omega_{1}\right)\right.\right. \\
& \left.+m\left(\Omega_{1}\right) \Delta \sigma\left(\Omega_{1}-\Omega_{2}\right)\right] \\
& +\frac{\Gamma a_{1}}{2}\left[m\left(-\Omega_{2}\right) \Delta \sigma^{2}\left(\Omega_{1}\right)\right. \\
& \left.+2 m\left(\Omega_{1}\right) \Delta \sigma\left(\Omega_{1}\right) \Delta \sigma\left(-\Omega_{2}\right)\right] \\
& +\Xi_{1}\left[\Delta \sigma\left(2 \Omega_{1}\right) \Delta \sigma\left(-\Omega_{2}\right)\right. \\
& \left.+\Delta \sigma\left(\Omega_{1}-\Omega_{2}\right) \Delta \sigma\left(\Omega_{1}\right)\right] \\
& \left.+\frac{X i_{2}}{2} \Delta \sigma^{2}\left(\Omega_{1}\right) \Delta \sigma\left(-\Omega_{2}\right)\right\}
\end{aligned}
$$

In the above expressions, $H(\Omega)=T(\Omega) / T(0)$ is the normalized XGM wavelength conversion transfer function, with $T(\Omega)$ given by (11) of [5].

From (1) and (6)-(9) closed expressions for the harmonic distortion and intermodulation products in the converted signal can be derived, resulting

$$
H D=\left|\frac{\Delta P_{2}\left(2 \Omega_{1}\right)}{\Delta P_{2}\left(\Omega_{1}\right)}\right|^{2}=\left|\frac{\Delta \sigma\left(2 \Omega_{1}\right)}{\Delta \sigma\left(\Omega_{1}\right)}+\frac{\Gamma a_{2}}{2} \Delta \sigma\left(\Omega_{1}\right)\right|^{2}
$$

$I M_{2}=\left|\frac{\Delta P_{2}\left(\Omega_{1} \Omega_{2}\right)}{\Delta P_{2}\left(\Omega_{1}\right)}\right|^{2}=\left|\frac{\Delta \sigma\left(\Omega_{1}-\Omega_{2}\right)}{\Delta \sigma\left(\Omega_{1}\right)}+\Gamma a_{2} \Delta \sigma\left(-\Omega_{2}\right)\right|^{2}$

$$
\begin{aligned}
I M_{3}= & \left|\frac{\Delta P_{2}\left(2 \Omega_{1}-\Omega_{2}\right)}{\Delta P_{2}\left(\Omega_{1}\right)}\right|^{2} \\
= & \mid \frac{\Delta \sigma\left(2 \Omega_{1}-\Omega_{2}\right)}{\Delta \sigma\left(\Omega_{1}\right)}+\frac{\Gamma^{2} a_{2}^{2}}{2} \Delta \sigma\left(\Omega_{1}\right) \Delta \sigma\left(-\Omega_{2}\right) \\
& +\left.\Gamma a_{2}\left[\frac{\Delta \sigma\left(2 \Omega_{1}\right) \Delta \sigma\left(-\Omega_{2}\right)}{\Delta \sigma\left(\Omega_{1}\right)}+\Delta \sigma\left(\Omega_{1}-\Omega_{2}\right)\right]\right|^{2} .
\end{aligned}
$$

Equations (6)-(12) are the main results of this letter and can find useful applications in the analysis of intermodulation distortion 


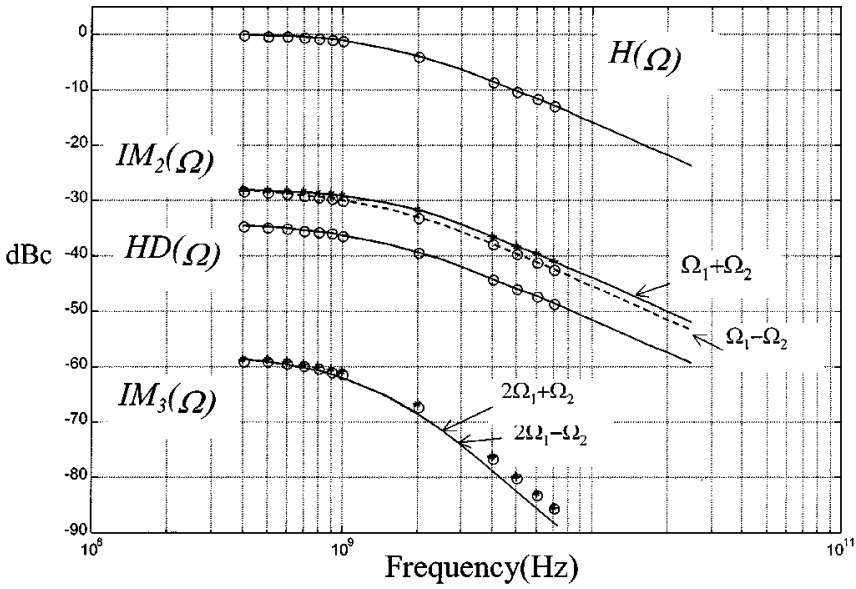

Fig. 2. Spectral dependences of the normalized linear transfer function, harmonic and intermodulation products of a two tone SCM input signal upon wavelength conversion via XGM in a SOA with typical parameters. Analytic results in solid traces. Numerical results shown in circles and stars.

in XGM wavelength converted SCM signals and in the evaluation of the role that the different parameters have on the signal impairment and therefore the system design. For instance, Fig. 2 shows the spectral variation of the above magnitudes (10)-(12) for a SOA based XGM converter with parameters for the SOA given by: $P_{\text {sat } 1}=P_{\text {sat } 2}=10 \mathrm{dBm}, \tau_{s}=340 \mathrm{ps}, a_{1}=a_{2}=$ $2.5 \times 10^{-16} \mathrm{~cm}^{2}, \Gamma=0.3, N_{1}=N_{2}=0.9 \times 10^{18} \mathrm{~cm}^{-3}$, $V=2.47 \times 10^{-10} \mathrm{~cm}^{3}, I=250 \mathrm{~mA}$, and signal parameters given by: $\bar{P}_{01}=\bar{P}_{02}=1 \mathrm{~mW}, m\left( \pm \Omega_{1}\right)=m\left( \pm \Omega_{2}\right)=0.05$ and $2 \pi\left(\Omega_{2}-\Omega_{1}\right)=100 \mathrm{MHz}$. For the sake of comparison, equivalent results obtained by numerical solution of (1)-(3) are also depicted, showing, as expected, excellent agreement. As it can be appreciated, both harmonic and intermodulation distortions show lowpass characteristics, however, with different roll-off frequencies than the linear normalized transfer function , which is also shown for reference. It should be pointed out as well, that these results indicate that potential high levels of inter- modulation distortion might appear in a wavelength converted version of a complete frequency plan of a SCM signal.

\section{SUMmARY AND CONCLUSION}

We have derived closed formulas for the harmonic and intermodulation distortion that appear on a wavelength converted two-tone SCM signal via XGM. These expressions can be useful for the design of systems and networks where WDM channels carrying SCM signals experience wavelength conversion. These results indicate that potential high levels of intermodulation distortion might appear in a wavelength converted version of a complete frequency plan of a SCM signal. For instance, in a standard NTSC frequency plan IMD values must be (for the worst case) below $-55 \mathrm{dBc}$. The values obtained here for a simple case of two tones although smaller, are close enough to envisage that problems will arise when the complete contribution of the frequency plan is accounted for. Further research on the applicability of wavelength conversion to SCM signals is required. In due course new results will be reported.

\section{REFERENCES}

[1] S. J. B. Yoo, "Wavelength converson technologies for WDM network applications," J. Lightwave Technol., vol. 14, pp. 955-966, 1996.

[2] T. Durhuus, B. Mikkelsen, C. Joergensen, S. L. Danielsen, and K. E. Stubkjaer, "All optical wavelength conversion by semiconductor optical amplifiers," J. Lightwave Technol., vol. 14, pp. 942-954, 1996.

[3] W. Shieh, E. Park, and A. E. Willner, "All optical wavelength shifting of microwave subcarriers by using four wave mixing in a semiconductor optical amplifier," IEEE Photon. Technol. Lett., vol. 8, pp. 524-526, 1996.

[4] P. N. Freeman, N. K. Dutta, and J. Lopata, "Semiconductor optical amplifier for wavelength conversion in subcarrier multiplexed systems," IEEE Photon. Technol. Lett., vol. 9, pp. 46-49, 1997.

[5] D. Marcenac and A. Mecozzi, "Switches and frequency conversters based on cross-gain modulation in semiconductor optical amplifiers," IEEE Photon. Technol. Lett., vol. 9, pp. 749-751, 1997.

[6] K. Lau and A. Yariv, "Intermodulation distortion in a directly modulated semiconductor injection laser," Appl. Phys. Lett., vol. 45, pp. 1034-1036, 1984.

[7] J. Helms, "Intermodulation distortions of broad-band modulated laser diodes," J. Lightwave Technol., vol. 10, pp. 1901-1906, 1992. 